\title{
A review of the electronic and magnetic properties of tetrahedrally bonded half-metallic ferromagnets
}

\author{
Ph Mavropoulos† and I Galanakis $\ddagger$ \\ † Institut für Festkörperforschung, Forschungszentrum Jülich, D-52425 Jülich, \\ Germany \\ $\ddagger$ Department of Materials Science, School of Natural Sciences, University of \\ Patras, Patras 265 04, Greece \\ E-mail: ph.mavropoulos@fz-juelich.de, galanakis@upatras.gr
}

\begin{abstract}
The emergence of the field of spintronics brought half-metallic ferromagnets to the center of scientific research. A lot of interest was focused on newly created transition-metal pnictides (such as $\mathrm{CrAs}$ ) and chalcogenides (such as $\mathrm{CrTe}$ ) in the metastable zinc-blende lattice structure. These compounds were found to present the advantage of high Curie temperature values in addition to their structural similarity to semiconductors. Significant theoretical activity has been devoted to the study of the electronic and magnetic properties of these compounds in an effort to achieve a better control of their experimental behaviour in realistic applications. This review is devoted to an overview of the studies of these compounds, with emphasis on theoretical results, covering their bulk properties (electronic structure, magnetism, stability of the zincblende phase, stability of ferromagnetism) as well as low-dimensional structures (surfaces, interfaces, nanodots and transition-metal delta-doped semiconductors) and phenomena that can possibly destroy the half-metallic property, like structural distortions or defects.
\end{abstract}

PACS numbers: $75.47 . \mathrm{Np}, 71.20 . \mathrm{Be}, 71.20 . \mathrm{Lp}$ 


\section{Contents}

1 Introduction 2

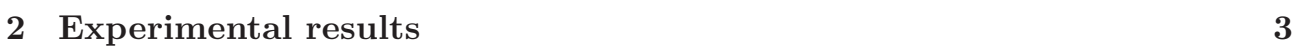

\begin{tabular}{|lll}
3 & Calculations on TM-based zinc-blende compounds & 5
\end{tabular}

3.1 Band structure and DOS . . . . . . . . . . . . . 5

3.2 Magnetic moments . . . . . . . . . . . . . . . . . . . 7

3.3 Local moment trends with cation valence and electronegativity . . . . 8

3.4 Effect of lattice parameter on half-metallicitv . . . . . . . . . . . . . 9

3.5 Effect of spin-orbit coupling on the polarization . . . . . . . . . . 10

4 Magnetic ground state, exchange interactions, and Curie temperature 11 4.1 Total energv results . . . . . . . . . . . . . . . . 12

4.2 Exchange interactions in the ground state and calculations of $T_{d} \ldots 12$

$\begin{array}{lll}5 & \text { Stability of the zinc-blende phase } & 13\end{array}$

$\begin{array}{lll}6 & \text { Surface half-metallicity } & 15\end{array}$

$\begin{array}{lll}7 & \text { Interfaces with semiconductors } & 16\end{array}$

7.1 Delta-doped zinc-blende semiconductors . . . . . . . . . . . . . . 17

$7.2 \quad$ Ab-initio interface engineering $\ldots \ldots \ldots \ldots \ldots$

$\begin{array}{lll}8 & \text { Effect of disorder } & 18\end{array}$

$\begin{array}{lll}9 & \text { Other tetrahedrally bonded structures } & 19\end{array}$

9.1 TM-based wurtzite compounds . . . . . . . . . . . . . . . . . . 19

9.2 Nanoclusters and nanodots . . . . . . . . . . . . . . . . . . . . . 19

10 Recent results on the role of electron correlations and on the temperature dependence of the polari

$\begin{array}{ll}11 \text { Summarv and outlook } & 21\end{array}$

\section{Introduction}

The rapid advance of magnetoelectronics and spintronics in recent years has given a strong boost in the search for novel magnetic materials, as are the half-metallic ferromagnets. These are spin polarized materials, which exhibit the property of having a metallic density of electron states for the one spin direction (usually majority spin), while there is a band gap around the Fermi level, $E_{F}$, for the states of the opposite spin. This half-metallic property (the name coined by de Groot and collaborators [1]) makes the one spin channel conducting, while the other is insulating (or semiconducting), so that, in the ideal case, half-metals can conduct a current which is $100 \%$ spin polarized.

The spin polarization $P$ at $E_{F}$ is expressed in terms of the spin-up and spin-down density of states, $n_{\uparrow}\left(E_{F}\right)$ and $n_{\downarrow}\left(E_{F}\right)$, as

$$
P=\frac{n_{\uparrow}\left(E_{F}\right)-n_{\downarrow}\left(E_{F}\right)}{n_{\uparrow}\left(E_{F}\right)+n_{\downarrow}\left(E_{F}\right)} \text {. }
$$


For an ideal half-metallic ferromagnet, $P=1$; however, a number of factors can reduce $P$ to lower values: defects and disorder, spin-orbit coupling, temperature, interface and surface states in the gap, etc. Apart from a polarization value as close as possible to unity, half-metals must have also other properties in order to be functional: a high Curie point $T_{C}$ and compatibility with semiconductors are among the key points for spintronics applications.

In 2000, Akinaga and collaborators 2] reported growth of a few layers of CrAs in the metastable zinc-blende phase $(\mathrm{zb})$ for the first time; this was achieved by molecular beam epitaxy on a GaAs substrate (the ground state structure of CrAs is the $\mathrm{MnP}$ structure [3]). In the zb phase, CrAs was reported to be ferromagnetic, with a Curie point higher than $400 \mathrm{~K}$. In the same publication, first-principles calculations of bulk zinc-blende CrAs showed the material to be a half-metallic ferromagnet; the moment per formula unit was calculated to be $3 \mu_{B}$, in agreement with the experimental result.

These findings initiated a strong activity, because several merits were combined: the half-metallic property, coherent growth on a semiconductor, and $T_{C}$ higher than room temperature. The activity was extended beyond zb CrAs, encompassing a variety of tetrahedrally bonded transition metal (TM) compounds with $s p$ atoms of the IV, V and VI groups of the periodic table. In this paper we review relevant investigations up to date, with emphasis on the theoretical results on the electronic structure and magnetic properties of these compounds. We do not extend our review to the related but vast topics of diluted magnetic semiconductors or other types of half-metals. We also note in passing the surprising (calculated) appearance of half-metallic ferromagnetism in tetrahedrally bonded compounds of I-V and II-V elements [4, 5, 6], such as zb CaAs, where no transition metals are involved. The origin of ferromagnetism is therefore of a different nature here, but a discussion of these compounds is beyond the purpose of the present work.

The remainder of the paper is summarized as follows. We begin with a summary of experimental findings in section 2 Section 3 is devoted to an analysis of the electronic and magnetic structure of half-metallic zinc-blende compounds, as obtained by firstprinciples calculations. Investigations on the magnetic ground state (ferromagnetic vs. antiferromagnetic), exchange interactions and Curie temperatures of such compounds are reviewed in section 4 The metastability of the zinc-blende phase, compared to the NiAs or other phases, is addressed in section [5 The possibility of surface halfmetallicity is discussed in section [ 6 In section 7 we review the properties of the interfaces with semiconductors, including delta-doping and transport in multilayered structures. We continue with a discussion on the effect of disorder on the half-metallic gap in section 8 Section 9 is devoted to tetrahedrally bonded TM-sp compounds with other structures, such as the wurtzite structure. Finally, in section [10 review results of calculations beyond density-functional theory. We conclude with a summary in section 11

\section{Experimental results}

Experimentally, CrAs in the zinc-blende structure was first grown by Akinaga et al. 2], by molecular beam epitaxy (MBE) on a GaAs substrate. The zb phase could be stabilized only for thin CrAs layers; for samples above a critical thickness of $3 \mathrm{~nm}$, the reflection high-energy electron diffraction (RHEED) pattern indicated an unknown different phase; such small thicknesses are typical in all experiments involving zb pnictides (compounds including group- $\mathrm{V}$ atoms) or chalcogenides 
(compounds including group-VI atoms). The measured saturation magnetization at low temperature corresponded to $3 \mu_{B}$ per formula unit, in good agreement with $a b$ initio calculations (which also predicted a half-metallic band structure). The precise Curie temperature was not reported, but it was found to be higher than $400 \mathrm{~K}$, suggesting applicability at room temperature. The surface morphology and magnetic characteristics of zb CrAs films under different growth conditions were studied in reference [7]. In reference [8], a thickness-dependence angular resolved photoemission spectroscopy (ARPES) study showed a dispersion for a $2 \mathrm{~nm}$ CrAs film, which was not present for a $30 \mathrm{~nm}$ film; this, together with the RHEED pattern, suggested a monocrystalline sample at $2 \mathrm{~nm}$, changing to polycrystalline at $30 \mathrm{~nm}$. ARPES data on a thin zb-CrAs film were also presented in reference [10.

Further work [1], based on fluorescence extended X-ray absorption fine structure (EXAFS), provided more evidence on the existence of zb phase on a $2 \mathrm{~nm} \mathrm{zb} C r A s$ film, and found a CrAs bond length of $2.49 \AA$ (in close agreement with the value of $2.52 \AA$ which is deduced from ab-initio calculations [12). Moreover, high-resolution transmission electron micrography (HRTEM) and X-ray grazing incidence diffraction (GID) were used in reference [13 to identify $2.5 \mathrm{~nm}$ ( 9 monolayers (ML)) thick zb CrAs on GaAs; it was found that the zb-phase can be partly deformed by appropriate annealing. The samples were ferromagnetic, the saturation magnetic moment of both phases was found to be about $3 \mu_{B}$ per formula unit, and $T_{C}$ was above $400 \mathrm{~K}$.

In parallel, zb CrAs/GaAs multilayers were grown by use of MBE and studied in references [9] and [14. In both studies the optimal (for the zb-structure) multilayers consisted of a period of $2 \mathrm{ML}$ of GaAs and $2 \mathrm{ML}$ of CrAs. The period was repeated 20 times in 9] and 100 times in [14. Magnetization measurements in [14 showed a magnetic moment of $2 \mu_{B}$ per formula unit of CrAs (lower than the theoretical prediction of $3 \mu_{B}$ ), and a Curie temperature of $800 \mathrm{~K}$.

$\mathrm{CrSb}$ was also grown by MBE in ultrathin films of a few monolayers in the zincblende structure [15] [16. In the work of reference [15], a GaAs, GaSb, and (Al,Ga)Sb substrates were used. The structure was examined by HRTEM and RHEED. The samples were ferromagnetic with a magnetic moment per formula unit reported to be between 3 and $5 \mu_{B}$ (the theoretical prediction is $3 \mu_{B}$ [17, 18, 19]) and a $T_{C}$ definitely exceeding $400 \mathrm{~K}$. In reference [16] it was shown how the substrate can be tailored for growth of somewhat thicker films of CrSb. While the maximal thickness on GaAs was found to be $1 \mathrm{~nm}(3 \mathrm{ML})$, an (In,Ga)As substrate provides a larger lattice parameter which can be tuned by changing the In composition to favor the growth of zb-CrSb. On an $\mathrm{In}_{0.08} \mathrm{Ga}_{0.92} \mathrm{As}$ substrate, $6 \mathrm{ML}$ of zb-CrSb were successfully grown, and for an $\mathrm{In}_{0.13} \mathrm{Ga}_{0.87}$ As substrate, growth of a $9 \mathrm{ML}(3 \mathrm{~nm}) \mathrm{zb}-\mathrm{CrSb}$ film could be achieved.

Also $\mathrm{CrSb} / \mathrm{GaAs}$ multilayers in the zb structure were grown [20]. Here, the $\mathrm{CrSb}$ thickness was just $1 \mathrm{ML}$, while the GaAs layers were $5 \mathrm{~nm}$ thick, and the multilayers included up to four periods. The zb-structure was monitored by RHEED measurements during growth, and cross-checked for dislocations at the interfaces by HRTEM. The saturation magnetization at low temperatures was close to the theoretical value of $3 \mu_{B}$. The ferromagnetic transition temperature was reported to be above $400 \mathrm{~K}$ (measurements at high temperatures were limited by the superconducting quantum interference device (SQUID)).

MnAs could be grown in nanodots in the zinc-blende structure [10, 21, 22 $\neq$.

$\ddagger$ In $[10$ it is reported that MnSb dots could not be directly grown in the zb-phase on GaAs, probably due to lattice mismatch. 
References [10] and 21] report growth of zb-MnAs dots of average size of about $16 \mathrm{~nm}$ on sulphur-terminated $\operatorname{GaAs}(001)$ surfaces, with a density of dots of $2.3 \times 10^{10} / \mathrm{cm}^{2}$. When the growth was continued, the MnAs dots changed to the NiAs structure (which is the equilibrium structure of MnAs). SQUID measurements showed the zb-MnAs dots to be ferromagnetic, with a Curie temperature of about $280 \mathrm{~K}$. Photoemission spectra 10, 21] show a localization of the Mn $d$ states, with absence of a Fermi edge§, a main peak at around $4 \mathrm{eV}$ below $E_{F}$, and a satellite at $6 \mathrm{eV}$ below $E_{F}$; these features are similar to $(\mathrm{Ga}, \mathrm{Mn})$ As diluted magnetic semiconductors, and quite different than MnAs in the NiAs structure, where a Fermi edge is clearly seen in photoemission [10, 21]. Similar are the conclusions of reference [10, where it is reported that, with increasing dot density (above $3 \times 10^{12} / \mathrm{cm}^{2}$ ), a Fermi edge evolves in the photoemission spectrum. This onset of metallic behaviour is attributed by the Authors either to formation of a NiAs-type of structure or to percolation among the clusters. We also note the possibility of delta-doped GaAs with Mn 23, 24]; see section 7.1] for further discussion.

Further experimental work on zinc-blende pnictides and chalcogenides includes MBE growth of $\mathrm{GaSb}(25 \AA) / \operatorname{MnSb}(2 \AA)$ (001) multilayers 25] and $\mathrm{CrTe}$ on $\mathrm{GaAs}(001)$ thin films [26. In both cases it is reported that there are strong indications of a $\mathrm{zb}$ phase, but no definite proof.

Finally, we note that, to our knowledge, the half-metallic property itself has not been experimentally proven or disproven in these compounds.

\section{Calculations on TM-based zinc-blende compounds}

\subsection{Band structure and DOS}

The principal mechanism leading to the appearance of the half-metallic gap in zincblende compounds of transition metals (TM) with $s p$ elements is the hybridization of the $d$ wavefunctions of the TM with the $p$ wavefunctions of the $s p$ atom. This $p$-d hybridization is dictated by the tetrahedral environment, where each atom is surrounded by four atoms of the other species. In particular, in the presence of tetrahedral symmetry the $d$-states split in two irreducible subspaces: the threefolddegenerate $t_{2 g}$ subspace, consisting of $d_{x y}, d_{y z}$ and $d_{x z}$ states, and the twofolddegenerate $e_{g}$ subspace, which includes the $d_{x^{2}-y^{2}}$ and $d_{z^{2}}$ states. Only states of the former subspace can hybridize with the $p$ states of the $s p$-atom neighbours, forming bonding and antibonding hybrids; the $e_{g}$ states, on the contrary, remain rather nonbonding. The situation is shown schematically in figure (up) for both spins, with the majority (spin-up) $d$ states being lower in energy than the spin-down states due to the exchange splitting.

Starting from this picture, we can proceed now to a discussion of the energy bands, shown in figure 1(down), and calculated within the local spin density approximation (LSDA) of density-functional theory (the As $4 s$ states are much lower in energy and omitted in the figure). We note that the notations $e_{g}$ and $t_{2 g}$ are, strictly speaking, valid only for states at the center of the Brillouin zone; however, the energy bands formed by the $e_{g}$ and $t_{2 g}$ states are energetically rather separated, therefore we keep this notation for the bands formed by these states. The strong $p$ - $d$ hybridization leads

$\S$ This absence of a Fermi edge, indicating an insulating phase, is not found by ab-initio calculations. In 10] 21 it is speculated that the reason is strong electron correlations, which are not well captured by local density-functional theory. 


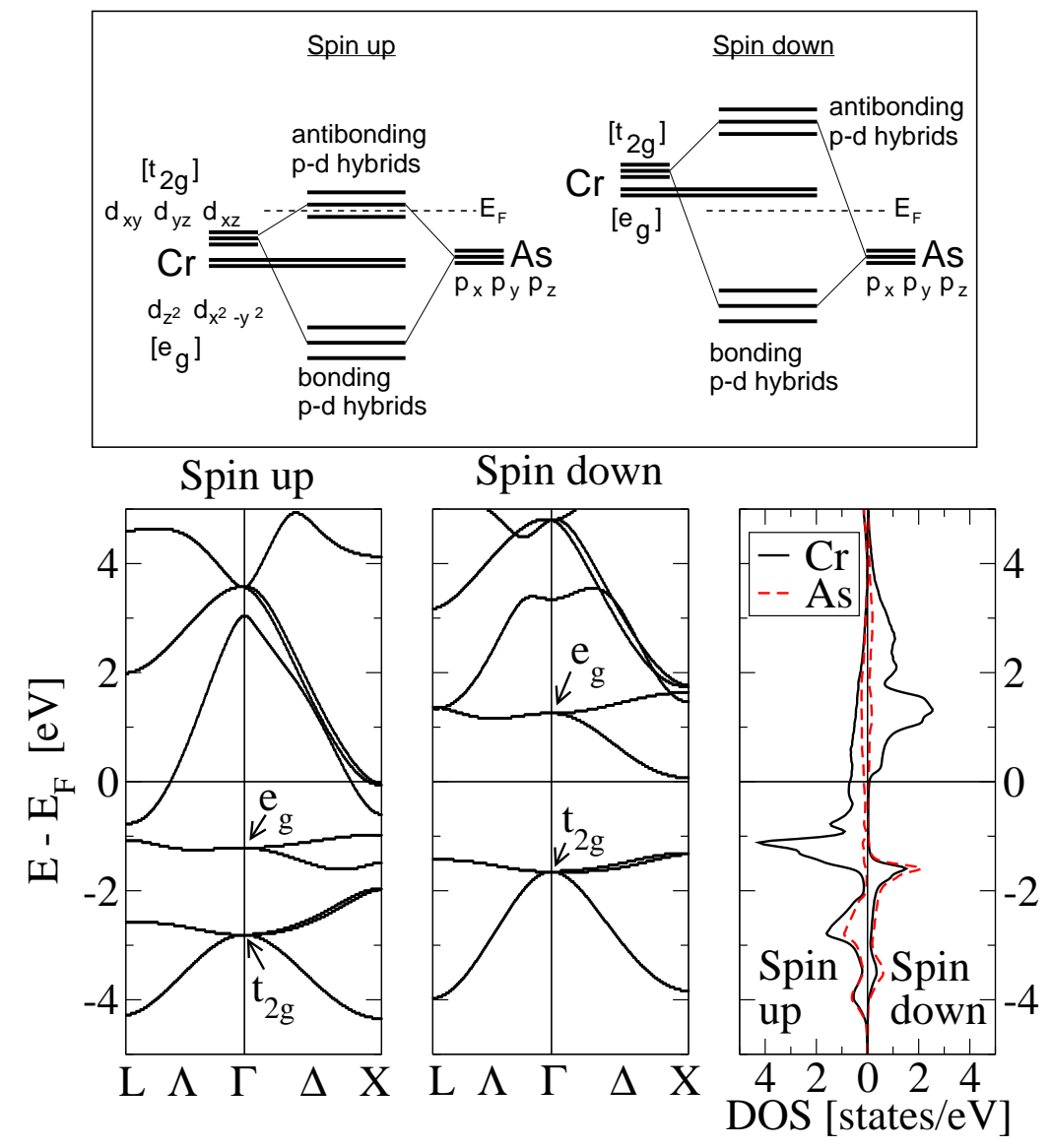

Figure 1. Up: Schematic description of the $p$ - $d$ hybridization leading to the halfmetallic property. Down: Calculated spin-resolved band structure and spin- and atom-resolved density of states of zinc-blende CrAs in the GaAs lattice parameter within the LSDA.

to wide corresponding $t_{2 g}$ energy bands, located, for spin up, at about -5 to $-2 \mathrm{eV}$ (bonding states), and above $E_{F}$ (antibonding); the latter are in the same energy range with the $\mathrm{Cr} s$ states. The rather non-bonding $e_{g}$ states, on the contrary, form mainly narrow bands. These remain energetically between the bonding and antibonding $p$ - $d$ bands, at about $-1 \mathrm{eV}$. For spin down, the situation is similar, with all bands shifted to higher energies because of the exchange splitting; the spin-down bands are also wider, because they reside at higher energies. The striking effect is that, for spindown, the exchange splitting is strong enough to push the $e_{g}$ states just above the Fermi level, so that $E_{F}$ is in the gap. This is also seen in the atom- and spin-resolved density of states (DOS). According to the calculated DOS, the material is manifestly half-metallic in its electronic and magnetic ground state.

This basic form of the electronic density of states is found by all relevant electronic structure calculations, within either the LSDA or the generalized gradient approximation (GGA) to density-functional theory. Calculations within the GGA show a slightly stronger local magnetic moment at the $\mathrm{Cr}$ site, resulting in a stronger 
exchange splitting and a wider gap; then, $E_{F}$ is even deeper within the gap. It is generally expected that the GGA yields a more accurate prediction of the lattice parameter and magnetic moment in magnetic compounds, with corrections of the order of $2-3 \%$.

The $p$ - $d$ hybridization, essential to the formation of the gap, is well-known as $p$-d repulsion in the physics of TM defects in semiconductors of zinc-blende (where the TM substitutes the cation) or diamond structure [27], and is essentially present also in diluted magnetic semiconductors (DMS) 28. In this respect, the electronic structure of zinc-blende pnictides and chalcogenides can be viewed as the electronic structure of TM-doped semiconductors in the limit of high concentration. From the electronic structure point of view, it is expected that all zinc-blende binary compounds of TM with group IV, V, and VI $s p$-atoms are candidates for the appearance of halfmetallic ferromagnetism - in fact, their calculated density of states is similar to the one of CrAs (e.g. see references [2, 19, 29, 30, 31, 32, 33, 34, 35, 36, 37, 38, 39]), with spin-down gaps around $0.5-1 \mathrm{eV}$ when the $s p$ element belongs to the IV-group, around $1-2 \mathrm{eV}$ when it belongs to the $\mathrm{V}$-group, and $1.5-2.5 \mathrm{eV}$ when it belongs to the VI-group, and with the spin-up bands appropriately shifted to achieve charge neutrality.

The half-metallic state is, however, not guaranteed in all of these compounds, nor is a ferromagnetic ground state. We discuss this in sections 4 and 5 As we shall see, other factors (lattice constant, antiferromagnetic interactions, structural instabilities, etc.) limit this expectation, but a number of compounds remains within these limits.

\subsection{Magnetic moments}

In the bulk of half-metallic compounds, the spin moment per formula unit is an integer (in $\mu_{B}$ ). Since $E_{F}$ is in the gap, the total number of spin-down valence electrons per formula unit, $N_{\text {tot }}^{\downarrow}$, is an integer. The total number of valence electrons per formula unit, $Z_{\text {tot }}$, must also be an integer, so it follows that the number of valence spin-up electrons, $N_{\text {tot }}^{\uparrow}=Z_{\text {tot }}-N_{\text {tot }}^{\downarrow}$, is an integer. Thus the magnetic moment, $M=\left(N_{\text {tot }}^{\uparrow}-N_{\text {tot }}^{\downarrow}\right) \mu_{B}=\left(Z_{\text {tot }}-2 N_{\text {tot }}^{\downarrow}\right) \mu_{B}$, is an integer. This is a necessary, but not sufficient, condition for half-metallicity. (At surfaces or interfaces this can change; see sections 6 and 7 )

Due to the similarity in the DOS of all zinc-blende pnictides and chalcogenides, the number of spin down valence states up to the gap is always four: there is the low-lying $s$ band of the $s p$ atom, and the three bonding $p$ - $d\left(t_{2 g}\right)$ bands. Therefore, assuming half-metallic behaviour, the total moment per formula unit follows a SlaterPauling-like "rule of 8":

$$
M_{\text {tot }}=\left(Z_{\text {tot }}-8\right) \mu_{B} .
$$

Analogous relations hold for the half-metallic Heusler alloys, where there is a SlaterPauling rule of 18 (in half-Heusler alloys) and rule of 24 (in full-Heusler alloys) [40. Thus, in the half-metallic state, CrAs and CrSb have a moment of $3 \mu_{B}$, while CrSe or MnAs (having one more valence electron) show a moment of $4 \mu_{B}$. Once more an analogy to the diluted magnetic semiconductors can be seen: calculations show that, for instance in $\mathrm{Ga}_{1-x} \mathrm{Mn}_{x} \mathrm{As}$, the spin moment per Mn atom is exactly $4 \mu_{B}$. Note that also ternary compounds, such as (Cr,Mn)As, follow the same rules, as long as all TM (here $\mathrm{Cr}$ and $\mathrm{Mn}$ ) occupy the same sublattice of the zinc-blende structure; for instance, see reference [1] for calculations on half-metallic CrAs/MnAs(001) superlattices. 
From an alternative viewpoint, we can say that the $p$ states, low-lying as they are in energy, act as reservoirs, hosting electrons which are donated by the TM until the $p$ states are filled up with 6 electrons. The remaining TM $d$ electrons build up the spin moment. This viewpoint neglects the essential $p$ - $d$ hybridization, but is useful for electron counting.

Similar to the pnictides and chalcogenides is the electronic structure of zb TMcompounds with $\mathrm{Si}, \mathrm{Ge}$, and $\mathrm{Sn}$ 31, 42, 43, 44. However, zb TM-compounds with $\mathrm{C}$ (e.g. $\mathrm{MnC}$ ) have a distinctly different density of states, with the half-metallic gap being among the spin-up states, while the spin-down bands are metallic [45, 46. This gives rise to a "rule of 12" instead of eq. (2), and originates from the huge gap induced by carbon 34 .

\subsection{Local moment trends with cation valence and electronegativity}

In terms of the $p$ - $d$ hybridization one can understand the DOS and local magnetic moment trends with changing cation valence and/or electronegativity. Firstly we note that the $s p$ atom has a moment oppositely oriented to the one of the TM; calculated values are shown in table 1 (up). To understand this, we observe that the spin-up $d$ states of the TM are lower in energy than the spin-down $d$ states; thus, when the $p$ - $d$ hybridization occurs, the bonding spin-up $t_{2 g}$ hybrids have a larger $d$ admixture and are more itinerant than the spin-down ones; the latter are more localized within the $s p$ atom. Thus, in the Wigner-Seitz cell of the $s p$ atom, the spin-down states are in the majority, leading to this "antiferromagnetic" coupling.

According to this picture, the following trends can be deduced under unchanged lattice parameter. On changing the $s p$ atom for a lighter one of the same group (therefore more electronegative), the $p$ states are shifted to lower energies and the spin-down $p$ - $d$ hybridization is reduced; the spin-down bonding $p$ - $d$ bands become more localized at the $s p$ atom, transferring to its vicinity spin-down charge from the TM. On the other hand, because of the proximity of the spin-up $d$ states, the spinup hybridization is reduced to a lesser extent (this can be seen by a tight-binding argument, as the hopping decreases with the energy separation). As a result, the local moment of the $s p$ atom increases; the same is true for the local moment of the TM, since the sum, $M_{\text {tot }}$, must be constant integer. Also the gap increases for lighter $s p$ atoms, because the $p$ states (forming the valence band) are shifted lower, and the $e_{g}$ states (forming the conduction band) are shifted higher by the stronger local TM moment and exchange splitting $\|$. Note also that TM-group VI compounds show a wider gap than TM-group V compounds, because the $p$ states of the former are lower in energy.

Competing with these effects is the strong reduction in lattice parameter due to the smaller volume of lighter $s p$ elements. This results in reduction of the local moments (due to stronger hybridization), and also loss of half-metallicity (as we discuss below); in table 1 (down) we show the effect of lattice compression on the local moments of CrAs. As it turns out, the effects of the change of lattice parameter by far outweigh the effects of electronegativity.

\| The exchange splitting is roughly $\sim I \cdot M$, where $M$ is the magnetic moment and $I$ is the exchange integral, having a value of about $0.9 \mu_{B} / \mathrm{eV}$ for transition metals. 


\begin{tabular}{rrrrrrrrr}
\hline Compound & CrN & CrP & CrAs & CrSb & MnAs & CrS & CrSe & CrTe \\
\hline$M(\mathrm{TM})$ & 3.89 & 3.32 & 3.27 & 3.15 & 4.07 & 3.86 & 3.83 & 3.75 \\
$M(s p)$ & -1.07 & -0.45 & -0.38 & -0.25 & -0.25 & -0.12 & -0.10 & -0.06 \\
\hline \hline $\mathrm{a}_{\text {latt }}(\AA)$ & 5.45 & 5.65 & 5.87 & 6.06 & 6.48 & & & \\
\hline$M(\mathrm{Cr})\left(\mu_{B}\right)$ & 2.59 & 3.02 & 3.15 & 3.27 & 3.55 & & & \\
$M(\mathrm{As})\left(\mu_{B}\right)$ & -0.13 & -0.20 & -0.29 & -0.38 & -0.60 & & & \\
\hline
\end{tabular}

Table 1. Up: Local moments (in $\mu_{B}$ ) of the TM and $s p$ atoms in zinc-blende pnictides and chalcogenides calculated within the LSDA in the (large) InAs lattice parameter (after reference 19), showing trends due to electronegativity of the anion. Down: Effect of changing the lattice parameter on the local magnetic moments of CrAs. The magnetization of the interstitial region (amounting to about half of the volume in the zb structure) is small and not included in the table.

\subsection{Effect of lattice parameter on half-metallicity}

We now turn to the effect of the lattice parameter on the half-metallic property. We begin by the observation that compression of the lattice shifts all energy levels higher, but has a greater effect on the extended $p$ states (and $p$ - $d$ hybrids), and a lesser effect on the localized $e_{g}$ states. Since $E_{F}$ is situated among the spin-up antibonding $t_{2 g}$ bands, under lattice compression $E_{F}$ will be carried to higher energies along with these levels; the position of the spin-down conduction band (which is of $e_{g}$ character) is less affected by compression. Therefore $E_{F}$ ultimately enters the $e_{g}$ conduction band and half-metallicity is lost. It also turns out that, under lattice expansion, $E_{F}$ enters the spin-down valence band, also causing loss of half-metallicity.

As a conclusion, zinc-blende chalcogenides and pnictides are half-metallic in a limited range of lattice parameters, and it is not guaranteed that the equilibrium lattice parameter $a_{\mathrm{eq}}$ is in this range. First principles calculations are needed in order to predict $a_{\mathrm{eq}}$ and the magnetic state and DOS at $a_{\mathrm{eq}}$. For practical purposes, a deviation from the calculated $a_{\mathrm{eq}}$ by a few percent is acceptable, allowing for a small error in the estimation of $a_{\mathrm{eq}}$ by density-functional calculations $\boldsymbol{\Phi}$, and for a slightly different lattice parameter of the substrate, on which the compound can grow pseudomorphically. We remind the reader that chalcogenides and pnictides are not at equilibrium in the zinc-blende structure, thus they can be grown only for a few monolayers on suitable substrates.

In table 22 we summarize results of LSDA calculations [19] of the half-metallic property at the lattice parameters of several semiconductors, together with the equilibrium lattice parameter, for several systems. Systems marked with a "+" are half-metallic, with a "-" are not half-metallic, while a " \pm " means that the system is at the edge of half-metallicity, with $E_{F}$ just touching the conduction band. Allowing for an LSDA underestimation of the lattice parameter, together with a small lattice mismatch with the semiconductor, we identify which systems can, in principle, be coherently grown (only for a few monolayers; see section 5 ) on which semiconductors, being at the same time half-metallic; we mark them with a box. In this way, we identify the following candidate combinations: VAs on GaAs, VSb on InAs and GaSb, $\mathrm{CrAs}$ on GaAs, CrSb on InAs and GaSb, MnSb on GaSb, VTe on ZnTe, CrSe on ZnSe and CdS, and CrTe on CdSe and ZnTe. Note that nitrides, phosphides and sulphides

I Especially the LSDA is known to underestimate, in general, $a_{\mathrm{eq}}$ by $2-3 \%$; GGA corrects this to a great extent, but in some systems it slightly can overestimate $a_{\text {eq }}$. 


\begin{tabular}{|c|c|c|c|c|c|c|c|c|}
\hline $\mathrm{a}(\AA)$ & GaN & $\operatorname{InN}$ & GaP & GaAs & $\mathrm{InP}$ & InAs & GaSb & $\mathrm{InSb}$ \\
\hline Compound & 4.51 & 4.98 & 5.45 & 5.65 & 5.87 & 6.06 & 6.10 & 6.48 \\
\hline (5.54) VAs & - & - & - & + & + & + & + & + \\
\hline (5.98) $\mathrm{VSb}$ & - & - & - & - & + & + & + & + \\
\hline$(5.52) \mathrm{CrAs}$ & - & - & - & + & + & + & + & + \\
\hline (5.92) $\mathrm{CrSb}$ & - & - & - & - & + & + & + & + \\
\hline$(5.36) \mathrm{MnAs}$ & - & - & - & - & + & + & + & + \\
\hline (5.88)MnSb & - & - & - & - & - & - & \pm & + \\
\hline $\mathrm{a}(\AA)$ & $\mathrm{ZnS}$ & ZnSe & CdS & CdSe & ZnTe & $\mathrm{CdTe}$ & & \\
\hline Compound & 5.41 & 5.67 & 5.82 & 6.05 & 6.10 & 6.49 & & \\
\hline$(5.56) \mathrm{VSe}$ & - & - & - & + & + & + & & \\
\hline (6.06) VTe & - & - & - & - & \pm & + & & \\
\hline (5.61) $\mathrm{CrSe}$ & - & \pm & + & + & + & + & & \\
\hline (6.07) CrTe & - & - & - & + & + & + & & \\
\hline
\end{tabular}

Table 2. Half-metallic property of several zinc-blende pnictides and chalcogenides at the experimental lattice parameters of several semiconductors. The calculated (LSDA) equilibrium lattice parameters are given in parentheses. Systems marked with a "+" are half-metallic, with a "_" are not half-metallic, while a " \pm " means that the system is at the edge of half-metallicity. A box indicates that a halfmetallic system has a suitable lattice parameter to grow on the corresponding semiconductor.

have very small lattice parameters and are not half-metallic; they are excluded from the table. Mn-group-VI compounds are not ferromagnetic, and also excluded.

\subsection{Effect of spin-orbit coupling on the polarization}

Spin-orbit coupling introduces a mixing of the two spin channels, so that the electron spin is not a good quantum number. Therefore, it is presence, it is expected that states which were previously accounted as spin-up states in the region of the half-metallic gap, will now have some contribution to the spin-down DOS; strictly speaking, the half-metallic property $(P=100 \%)$ can never be achieved, since some amount of spinorbit coupling is present in all materials. Then, the half-metallic gap turns into a pseudo-gap, with some spectral intensity.

However, first-principles calculations [47, 48, show that the effect can be small, of the order of $1 \%$, except in the case when heavy elements are present. Thus, when spin-orbit coupling is included in the calculations, CrAs shows a polarization of $P=99.6 \%$, CrSb shows $P=98.6 \%$ (note that $\mathrm{Sb}$ is heavier than $\mathrm{As}$ ), and $\mathrm{MnBi}$ shows a much reduced polarization of $P=77 \%$ (neglecting spin-orbit coupling, $\mathrm{MnBi}$ is predicted 32, 49, to be half-metallic). These results demonstrate that spin-orbit coupling is significant for a heavy element such as $\mathrm{Bi}$, also because of its $6 p$ states which are directly involved in the band structure around $E_{F}$. The calculated orbital moment of $\mathrm{MnBi}$ is large, too, reaching a value of $0.11 \mu_{B}$.

A theoretical analysis based on perturbation theory [47 shows that the spectral properties in the half-metallic spin-down pseudo-gap depend (i) on the spin-up DOS in the same region and (ii) on the proximity of the energy to the band edges. It is deduced (and verified by ab-initio calculations) that (i) the spin-down DOS is a weak reflection of the spin-up DOS, depending quadratically on the spin-orbit coupling 
spin-flip strength, $n_{\downarrow}(E) \sim\left|V_{\mathrm{so}}\right|^{2} n_{\uparrow}(E)$; and (ii) that, close to the band edges, $n_{\downarrow}(E)$ increases strongly, so that it must be treated beyond first order in perturbation theory (because of the degeneracy of unperturbed spin-up and spin-down eigenvalues at the band edge).

\section{Magnetic ground state, exchange interactions, and Curie temperature}

In the discussion up to now we assumed a ferromagnetic ground state, and experiment has shown that $\mathrm{CrAs}, \mathrm{CrSb}$, and $\mathrm{MnAs}$ in the zinc-blende phase are ferromagnetic (see section 21). However, the ferromagnetic ground state has to be attested by calculations for the not-yet-fabricated compounds. In a number of papers [12, 27, 31, 33, 37, 42, 50, 51, 52, 53, 54, 55, 56, this aspect is examined via firstprinciples calculations. In the framework of such calculations there are three ways to attest the ferromagnetic ground state. (i) Non-collinear magnetic calculations can be made, where the system is allowed to relax in any arbitrary non-collinear configuration. (ii) Total energy calculations can be made in the ferromagnetic and in several antiferromagnetic states (perhaps including disordered local moment states within the coherent potential approximation), seeking the energy minimum. (iii) Starting from a ferromagnetic state, the interatomic exchange constants can be calculated (usually assuming interactions within a Heisenberg model), from which conclusions can be drawn as regards the ground state, as well as excited state properties, including the Curie temperature (see section 4.2). These methods are to a great extent complementary, and their applicability depends on the size of the system as well as the computational method used. The most accurate way of calculation is probably (i), but it is often not practical because it requires use of large supercells, solution of equations coupling the two spin channels, and must be applied to many starting configurations, especially if there are more than one local energy minima. Thus, (ii) and (iii) are most common in practice, and can in most cases ascertain if the ground state is indeed ferromagnetic or not. We note, however, that if the magnetic ground state is not ferromagnetic, it is likely to be non-collinear, since the fcc geometry (corresponding to the TM sublattice) causes magnetic frustration in the case of antiferromagnetic exchange interactions.

As a general principle (but with notable exceptions, depending mainly on the lattice parameter), the magnetic ground state of TM pnictides and chalcogenides in the zinc-blende structure is ferromagnetic, if the total number of valence electrons $Z_{\text {tot }} \leq 12$. According to eq. [2 this means that the maximum moment in the halfmetallic state is $4 \mu_{B}$. Thus, for instance, MnAs is expected to be ferromagnetic, but MnSe or FeAs are expected to be antiferromagnetic (or non-collinear). The reasoning behind this rule is the following. Ferromagnetic order in these compounds is brought about by the double exchange mechanism (energy is gained by a band-broadening at $E_{F}$ due to hybridizations) involving the spin-up antibonding $t_{2 g}$ states. Once $Z_{\text {tot }}$ exceeds 12 , the spin-up $t_{2 g}$ states are fully occupied, and the double exchange mechanism is no more present (the band broadening does not bring any gain in energy); thus the ferromagnetic order is lost. ${ }^{+}$

+ The same cause of ferromagnetism has been identified in some TM-doped diluted magnetic semiconductors 28, although those systems are rather more complicated involving also a Zener $p-d$ exchange. 


\subsection{Total energy results}

In accordance with the above, total energy calculations 12 have shown that VAs, CrAs and MnAs are ferromagnetic, while FeAs was found to have an antiferromagnetic ground state (the possibility of a non-collinear state was not examined in [12]). The energy gain for the formation of the ferromagnetic state (compared to the antiferromagnetic) is about $0.2 \mathrm{eV}$ for VAs, $0.3 \mathrm{eV}$ for $\mathrm{CrAs}$, and $0.1 \mathrm{eV}$ for MnAs; the antiferromagnetic state of FeAs provides an energy gain of $0.1 \mathrm{eV}$ compared to the ferromagnetic state (all energies calculated within the LSDA) [12. Calculations on $\mathrm{MnTe}$ in the zinc-blende structure have shown that it also has an antiferromagnetic ground state 27. Other calculations have confirmed this aspect. Total energy calculations have confirmed a ferromagnetic ground state for MnAs [50], CrAs [33. 37 ] and $\mathrm{CrSb}$ [33, 37.

A recent study 56] on MnAs concludes that, as $E_{F}$ enters the spin-down conduction band, there occurs a Fermi surface nesting (i.e., a large area of the spindown Fermi surface is almost parallel to the spin-up Fermi surface, being separated by an almost constant vector $\vec{q}$ ), allowing for virtual spin-flip excitations of wavevector $\vec{q}$ and leading to a corresponding non-collinear state. From another viewpoint, it has been suggested [52, 57] that there are two factors determining the firstneighbour Mn-Mn interaction in MnAs: a direct $\operatorname{Mn}(d)-\operatorname{Mn}(d)$, antiferromagnetic interaction and an indirect ferromagnetic $\operatorname{Mn}(d)-\operatorname{As}(p)-\operatorname{Mn}(d)$ interaction. The former is dominant at close Mn-Mn distances, inducing an antiferromagnetic state, but as the lattice parameter increases the overlap of the $d$ states of neighbouring Mn atoms is lost, so that the indirect, ferromagnetic interaction dominates. This fragility of ferromagnetism in MnAs is in accordance to the results of exchange interaction studies (section 4.2).

\subsection{Exchange interactions in the ground state and calculations of $T_{C}$}

Sakuma 31] reports a systematic investigation of the interatomic exchange interactions in CrAs, MnSi, MnGe, and MnSn in the zinc-blende structure. Under the assumption of the validity of a Heisenberg model, with a Hamiltonian of the form

$$
H=\sum_{i j} J_{i j} \hat{e}_{i} \hat{e}_{j}
$$

(where $\hat{e}_{i}$ is the magnetic moment direction at site $i$ ), the exchange constants $J_{i j}$ are found via the Liechtenstein formula [58. In this formula, the ab-initio band structure properties enter, so that the exchange constants are calculated without any adjustable parameter. The coefficient $J_{0}=\sum_{i \neq 0} J_{0 i}$, corresponding to the band-energy cost for flipping the magnetic moment of a single atom, reflects a "single-site spin stiffness", and the ferromagnetic state is stable when $J_{0}>0$; a rotation of the local moment by a small angle $\delta \theta$ costs energy $\delta E=J_{0}(1-\cos \delta \theta)$, while the mean-field Curie temperature is $T_{C}^{\mathrm{mf}}=2 J_{0} / 3 k_{B}$ (with $k_{B}$ the Boltzmann constant). This method is not as accurate as a full self-consistent calculation of a magnetic moment flip, but it is much faster and efficient in showing trends.

All studied compounds in 31] were found to be ferromagnetic for a wide range of lattice parameters; $J_{0}$ increases as a function of lattice parameter, as $E_{F}$ enters deeper into the half-metallic gap. Furthermore, by treating $E_{F}$ as a parameter for a fixed band structure (and calculating $J_{0}\left(E_{F}\right)$ ), the relative contribution to the coupling at each energy was found as a function of band-filling. It was shown that the spin-up 
antibonding $t_{2 g}$ states contribute to the ferromagnetic stability, while the spin-down conduction band states $\left(e_{g}\right.$ and antibonding $\left.t_{2 g}\right)$ induce an antiferromagnetic coupling and cause a change of sign to $J_{0}$ as $E_{F}$ enters the conduction band. The highest values for $J_{0}$ are found for $E_{F}$ in the middle of the gap. The local-moment-flip energy cost in CrAs, in the GaAs lattice constant (and at the real $E_{F}$ ), was found to be about $0.3 \mathrm{eV}$, which compares well with the ferromagnetic-antiferromagnetic energy difference of $0.3 \mathrm{eV}$ calculated by Shirai 12 .

Interatomic exchange interactions were also studied in [37 for $\mathrm{V}, \mathrm{Cr}$, and Mn compounds with $\mathrm{As}, \mathrm{Sb}$, and $\mathrm{P}$. It was found that the strongest tendency for ferromagnetism is among the Cr-based compounds, a result verified in [42] for pnictides. In $\mathrm{CrAs}$, the first-neighbour $\mathrm{Cr}-\mathrm{Cr}$ exchange constant $J_{1}$ is dominant, of the order of $15 \mathrm{meV}$. The second-neighbor $\mathrm{Cr}-\mathrm{Cr}$ interaction $J_{2}$ is negative (antiferromagnetic) and an order of magnitude smaller (about $-0.1 \mathrm{meV}$ ); due to the half-metallic gap, the exchange constants fall off rapidly with distance.

In reference [42], the stability of ferromagnetism in zinc-blende CrAs, CrSe, MnAs, $\mathrm{MnC}, \mathrm{MnSi}$, and MnGe was studied, again based on the calculation of exchange constants. Similarly to reference [31, lattice compression was found to destabilize the ferromagnetic state, as $E_{F}$ approaches and enters the spin-down conduction band. The effect was found to be quite drastic in MnAs, with the ferromagnetic Mn-Mn interaction $J_{1}$ decreasing in magnitude, and the antiferromagnetic Mn-Mn interaction $J_{2}$ increasing in magnitude; this effect was even stronger in CrSe. (In the other compounds, the trends are similar, but not as drastic.) Thus, as the MnAs lattice parameter is reduced from $5.87 \AA$ to $5.68 \AA$ (a change of only about $3.5 \%$ ), the Curie temperature (calculated within the random phase approximation) $T_{C}^{\text {rpa }}$ drops from $551 \mathrm{~K}$ to $136 \mathrm{~K}$; and $\mathrm{CrSe}$, for a similar compression, is found to change phase from ferromagnetic to antiferromagnetic ( $T_{C}^{\mathrm{rpa}}$ becomes negative). The trend of the mean-field result, $T_{C}^{\mathrm{mf}}$, is similar. An inspection of the density of states leads to the conclusion that the antiferromagnetic susceptibility becomes dominant as $E_{F}$ enters the spin-down conduction band due to compression; this conclusion is in agreement with the result of Sakuma [31, as well as with the results reported by Kübler [51] and by Sanyal et al. [56].

Curie temperatures were calculated in references [37, 42 and [51. To this purpose, several methods have been employed. For the solution of the Heisenberg model (after calculation of the exchange constants by Brillouin-zone integration of static-spin-spiral energies), there have been used the Monte-Carlo method [37] (which are the most accurate), the mean-field approximation (mfa) 37, 42] (overestimating $T_{C}$ ), or the random-phase approximation [42] (rpa) (more accurate than the mfa). Alternatively, the approach in [51] identifies the non-local dynamic susceptibility including an adjusted damping parameter. Due to the different approaches in the various works, and without experimental results on $T_{C}$ in bulk systems, a direct comparison of the result for $T_{C}$ is meaningless, however a comparison of $T_{C}$-trends calculated within the same method is definitely meaningful. It is a common conclusion that the ferromagnetic state is most robust in CrAs, which shows the highest calculated value of $T_{C}$ (ranging between $790 \mathrm{~K}$ and about $1200 \mathrm{~K}$, depending on the method).

\section{Stability of the zinc-blende phase}

The bottleneck for the fabrication of chalcogenides and pnictides in the zinc-blende structure seems to be their structural instability. The ground state structure of these 
compounds is in many cases the hexagonal NiAs structure (MnP structure for $\mathrm{CrAs}$ ), in which they are not half-metallic. The zinc-blende phase is metastable, and needs therefore to be stabilized via pseudomorphic growth on a semiconductor substrate of the zinc-blende or diamond structure.

Total energy calculations [18, 34, 35, 36, 38, 49, 50, 59, 60, 61, 62, have confirmed that the NiAs structure has considerably lower energy than the zb structure for almost all TM pnictides and chalcogenides, with the exception of nitrides [53] 63] which are reported to have a rock-salt ground state (for the early transition metal compounds $\mathrm{ScN}-\mathrm{CrN}$ ) or a zinc-blende ground state (for the late transition metal compounds, $\mathrm{MnN}-\mathrm{CoN}$ ); the nitrides, however, have too small a lattice parameter to be half metallic, and are beyond the scope of our discussion. The bulk zinc-blende phase has typically a higher energy of the order of $\Delta_{\text {bulk }} \sim 0.3-1 \mathrm{eV}$ per formula unit than the NiAs phase (when both are calculated at their corresponding equilibrium lattice constants); the NiAs phase has also a considerably smaller equilibrium unit cell volume $V_{\text {eq }}$ (e.g., reference [59] reports for MnAs in the NiAs structure $V_{\text {eq }} \approx 31 \AA^{3}$ and $V_{\mathrm{eq}} \approx 45 \AA^{3}$ in the zinc-blende structure). It has been reported [35] that $\Delta_{\text {bulk }}$ is lower for chalcogenides ( $\mathrm{CrTe}$, CrSe, and VTe were considered, with $\Delta_{\text {bulk }} \sim 0.3$ to $0.5 \mathrm{eV}$ ) than for pnictides $\left(\Delta_{\text {bulk }} \sim 0.5\right.$ to $\left.1.0 \mathrm{eV}\right)$, possibly giving an advantage to the growth of chalcogenides in the zinc-blende phase.

Typically, under sufficient volume expansion, the NiAs phase becomes unfavourable compared to the zinc-blende phase (the energy curves cross, and $\Delta_{\text {bulk }}$ becomes negative at some increased lattice constant). However, more important than $\Delta_{\text {bulk }}$ is the epitaxial energy difference $\Delta_{\text {epi }}$ (i.e., the energy difference between the two phases at a particular lattice constant when also a change of the c/a ratio is allowed), since a compound growing epitaxially on a substrate of increased lattice parameter will be able to adjust its c/a ratio in favour of elastic energy. This was pointed out in reference 62, where a number of compounds were studied in this aspect (MnAs, CrAs, $\mathrm{CrSb}, \mathrm{CrS}, \mathrm{CrSe}$, and $\mathrm{CrTe}$ ). It was found that $\Delta_{\text {epi }}$ always favours the NiAs phase (for all hypothetical substrate lattice parameters), except for CrSe, for which $\Delta_{\text {epi }}$ becomes negative at approximately $6.2 \AA$, favouring an epitaxial growth in the zincblende phase for substrates of higher lattice constant. Nevertheless, these conclusions are valid for the growth of thick layers, while a few monolayers will be much affected by the interfacial energy. This agrees with the experimental observation that $\mathrm{CrAs}$ and $\mathrm{CrSb}$ can be grown in the zinc-blende phase for thicknesses up to a few monolayers, and then break up.

The stability of the zinc-blende phase has also been examined with respect to tetragonal and rhombohedral deformation 35, 49, 64, 65] 66 (starting from the equilibrium volume in the zinc-blende structure). It was found that, under rhombohedral deformation, chalcogenides are stable 35, 65, while, among the studied pnictides, only $\mathrm{CrAs}, \mathrm{CrSb}$, and MnAs are stable (although rather soft, with shear moduli of the order of 3-5 GPa) 65]. Tetragonal deformation was found to be favourable for $\mathrm{MnSb}$ and $\mathrm{MnBi}$ 49, with an equilibrium c/a 0.75 , but the halfmetallic character was not changed. This stability of the half-metallic character under tetragonal distortion in many TM pnictides and chalcogenides is the conclusion of a number of works 65, 49, 64, 65, 68, 67. In particular, a tetragonalization along the [001] direction (expected in the presence of a (001) interface) distinguishes the $z$ axis and causes a splitting of the $t_{2 g}$ and $e_{g}$ irreducible representations at the Brillouin zone center $\Gamma$ 68. The $t_{2 g}$ threefold degeneracy splits into a twofold degeneracy (involving the $d_{x z}-p_{y}$ and $d_{y z}-p_{x}$ hybrids) and a non-degenerate level (involving the 
$d_{x y}-p_{z}$ hybrids); the $e_{g}$ twofold degeneracy splits into two non-degenerate levels, one involving the $d_{z^{2}}$ levels and one involving the $d_{x^{2}-y^{2}}$ levels. These splittings affect the spectral function at $\Gamma$, but they are in no way important for the overall DOS and the gap.

However, in reference [66 it is reported that a number of compounds (including CrSe and MnAs) cannot be half-metallic even at large the substrate lattice constants, because of the unfavourable vertical relaxation and c/a ratio. An interesting conclusion of the same work is that, for some pnictides (including $\mathrm{CrAs}, \mathrm{MnAs}$, and $\mathrm{MnSb}$ ) the zinc-blende phase is unstable with respect to a "gliding" tetragonal deformation, where the in-plane lattice parameter changes with simultaneous change of the $\mathrm{c} / \mathrm{a}$ (so that the bond length is kept constant). In practice, however, a tetragonal deformation of this particular kind cannot take place, because the in-plane lattice parameter is fixed by the substrate.

\section{Surface half-metallicity}

The surfaces of half-metallic ferromagnets are not guaranteed to be also half-metallic. This is because wavefunction hybridizations and bonding-antibonding splittings, essential to the appearance of the gap, change significantly at the surface; the "missing neighbours" can result in the appearance of surface states in the gap, destroying half-metallicity. This is, for instance, the case in most surfaces of half-metallic Heusler alloys [69. Although half-metallic surfaces are not of particular technological interest (contrary to half-metallic interfaces), they are of value for proving the halfmetallic property by spin-polarized photoemission experiments, which are surface sensitive*. In zinc-blende pnictides and chalcogenides, it was demonstrated by $a b$ initio calculations [19, 70, 71, 72] that the (001) surfaces can, conditionally, be halfmetallic.

Surface half-metallicity in the (001) surfaces of these compounds can occur only if they are terminated with the TM. In case of termination with the $s p$ atom, dangling bonds appear within the half-metallic gap, destroying half-metallicity (the possibility of surface reconstruction was, however, not considered in the calculations in [19, 70]). Similar effects were found in reference $[73$.

In case of TM termination, the electronic structure and DOS changes at the surface (even if it is half-metallic). A simple electron counting shows that, due to the missing $s p$ neighbour, $\left(8-Z_{s p}\right) / 2$ additional electrons must be accommodated by the surface TM, where $Z_{s p}$ is the valency of the $s p$ atom. To understand this, consider that, in the bulk, these electrons would occupy bonding $p$ - $d$ hybrids; here, half of these hybrids are missing. Local charge neutrality requires that these electrons remain in the vicinity of the surface (mostly in the surface layer). Moreover, half-metallicity is only preserved if they occupy spin-up states (otherwise $E_{F}$ will enter the conduction band). Therefore, in the cases when half-metallicity is preserved, the spin moment at the surface increases by $\left(8-Z_{s p}\right) / 2 \mu_{B}$ per surface atom. This also leads to an increase of the exchange splitting, shifting the spin-down $e_{g}$ levels higher in energy, thus also increasing the gap width. Such is the calculated case in, e.g., CrAs, VAs, or CrSe. On the other hand, MnAs has a bulk moment of $4 \mu_{B}$ per formula unit. If its Mnterminated, (001) surface were to retain the half-metallic character, the local moment

* See, e.g., reference 102 on an experimental study on the (0001) surface of MnSb in the (non-halfmetallic) NiAs structure 
should increase to $5.5 \mu_{B}$. This, however, is not possible, since the spin-up $e_{g}$ and antibonding $t_{2 g}$ states can accommodate at most 5 electrons, leading to a maximum moment of $5 \mu_{B}$. As a result, $e_{g}$ spin-down states are occupied, and half-metallicity is lost.

Also the (110) surfaces of $\mathrm{CrP}$ and $\mathrm{CrAs}$ were found to be half-metallic in reference [74, 75] (the calculation of $\mathrm{CrP}$ [74] was done at an expanded lattice constant $(5.48 \AA)$, at which also bulk half-metallicity appears). Interestingly, the (110) surface contains both kinds of atoms ( $\mathrm{Cr}$ and $\mathrm{P}$ or $\mathrm{Cr}$ and $\mathrm{As})$, and no dangling bonds appear to destroy the half-metallic property.

Since, from the electronic structure point of view, all ferromagnetic zinc-blende pnictides and chalcogenides are similar, it is expected that surface half-metallicity is likely to appear in such compounds. However, more theoretical investigations are necessary to verify this, examining in particular the effect of lattice relaxations and surface reconstruction.

\section{Interfaces with semiconductors}

For technological applications of half-metals, especially in magnetic tunnel junctions, it is imperative to eliminate the interface states in the gap region (i.e., for spin down) of the half-metal-semiconductor interface. The reason is that these states can act as carrier reservoirs and contribute to the transport, although they are localized, because the tunneling rate can be much lower than the refill rate of these states by spin-orbit coupling or inelastic effects [76]. Contacts of half-metallic Heusler alloys with semiconductors are known to host such interface states in almost all studied cases [77. It is therefore gratifying that pnictides and chalcogenides in the zincblende structure retain their half-metallicity also at the interfaces with semiconductors (which are anyhow the natural substrate for growth of these half-metals). The reason is that the bonding character changes coherently at the interface. For example, at a $\mathrm{CrAs} / \mathrm{GaAs}$ interface (with an As interface layer), the $p$ states of the As interface atoms hybridize with the $\mathrm{Cr} d$-states on the one side and with the Ga $s$ states on the other. Thus, the bonding-antibonding splitting does not cease at the interface. Such a behaviour is expected also in view of half-metallicity in TM-doped diluted magnetic semiconductors [28.

Interfaces of half-metallic pnictides and chalcogenides in the zinc-blende structure with III-V and II-VI semiconductors (in the same structure) were studied theoretically in 68, 73, 178, 79, 80, 81, 82, 83. It was found that no interface states appear at $E_{F}$ within the half-metallic gap, because of the coherent bonding described above. Moreover, moderate tetragonalization (which is expected in the case of a slight lattice mismatch) was not found to alter the half-metallic character. (See section 5 for a discussion of tetragonalization).

The half-metallic gap was found to be persistent also in the case of a $50 \%$ itermixing at the interfaces [68, in the sence that, at CrAs/GaAs for instance, the interface layer is half occupied by Ga and half by Cr. This is expected, since, again, the bonding continues coherently, and it is known that half-metallicity is present for a wide range of concentrations in diluted magnetic semiconductors [28].

The gap remains present at the interface also in the case that the semiconductor and half-metal anions are different (e.g., $\mathrm{CrAs} / \mathrm{GaSb}$ ). If, however, the valency of the cation changes, the electronic structure is more complicated 68. As an example we take a $\mathrm{CrSb} / \mathrm{ZnTe}(001)$ interface, where $\mathrm{ZnTe}$ is a II-VI semiconductor, while $\mathrm{Sb}$ 
is a group $\mathrm{V}$ element. Interface states appear at $E_{F}$ in the case of direct Zn-Sb contact, i.e., if the layered structure is of the form ...CrSbCrSbZnTeZnTe... On the other hand, if the interface atoms in contact are $\mathrm{Cr}$ and $\mathrm{Te}$ (structure of the form ...CrSbCrTeZnTe...), the gap is preserved at the interface. In the latter case, there is also a local increase of magnetic moment at the interface $\mathrm{Cr}$ atoms from 3 to $3.5 \mu_{B}$ because the Te atoms have one less $p$ hole than the Sb atoms, thus the interface $\mathrm{Cr}$ spin-up charge is increased.

\subsection{Delta-doped zinc-blende semiconductors}

Delta-doping of semiconductors by TM constitutes a special, limiting case of halfmetal-semiconductor interface [17, 23, 44, 84. Such "digital" compounds are of special interest, because of their two-dimensional half-metallic behaviour and the highly anisotropic transport properties 84. Also, delta-doping is a possible way to overcome the low solubility limit of transition metals in III-V semiconductors, making such constructions interesting as a special case of diluted magnetic semiconductors.

Experiments on GaAs, delta-doped with Mn (in the submonolayer range), found the compound to be ferromagnetic 23]. The Curie temperature was dropping with the distance between the Mn delta-layers, but saturated for large distances, indicating ferromagnetism of each single layer. A two-dimensional ferromagnetic, half-metallic character was also found by calculations in GaAs delta-doped with Mn [17, 84. In reference [84, the conductivity was also calculated, and it was found that the in-plane conductivity was metallic (only for spin up), with the current confined at and close to the Mn layers, while the perpendicular-to-the-plane conductivity was extremely low, reflecting the tunneling between the $\mathrm{Mn}$ layers. It is known that ferromagnetic order in DMS (in particular $\mathrm{Ga}_{1-x} \mathrm{Mn}_{x} \mathrm{As}$ ) is assisted by the $p$ holes introduced by the Mn dopants (due to the $p$ - $d$ repulsion) 28. As Mn-delta-doped GaAs can be viewed as a limiting case of DMS, it is interesting to see 84] that also the $p$-holes are in this case confined at and close to the Mn layers. Calculations on a delta-doped layer of Mn in Si 44 and Ge 85] also showed two-dimensional half-metallic ferromagnetism, based on the same basic mechanism.

In reference 52, a MnAs/Si superlattice is calculated to induce antiferromagnetic order to MnAs, possibly due to the small lattice parameter; a small $2 \%$ tetragonal distortion (with increased $\mathrm{c} / \mathrm{a}$ ) restores the ferromagnetic state.

Concerning the exchange interactions, a study of the exchange constants in Mndelta-doped Ge and GaAs was presented in reference 86. It was found that the systems are ferromagnetic, dominated by $J_{1}>0 \sharp$, while $J_{2}<0$ is lower in magnitude and antiferromagnetic (as is the case in the bulk of zb compounds MnGe and MnAs 42). Since density-functional calculations underestimate the semiconductor gap, calculations were performed with an ad-hoc increased gap, in order to examine its effect on the exchange constants. On increasing the semiconductor gap (either for Ge or for GaAs), the calculations [86] showed the systems to become more "ferromagnetic", in the sense that $J_{1}$ increases while $J_{2}$ decreases in magnitude.

\subsection{Ab-initio interface engineering}

The ferromagnetic and antiferromagnetic properties of TM chalcogenides and pnictides can be applied to novel tunnel junctions. In reference [6], first principles calculations

$\sharp$ For the notation see section 4.2 
showed how one can design a tunnel junction of half-metallic CrTe elements which are antiferromagnetically coupled via a semiconducting CdTe spacer. The idea is to introduce a monolayer $\mathrm{Mn}$ at the $\mathrm{CrTe} / \mathrm{CdTe}$ interface, so that the junction is of the form ...( $\mathrm{CrTe})_{2} \mathrm{MnTe}-(\mathrm{CdTe})_{n}-\mathrm{MnTe}(\mathrm{CrTe})_{2} \ldots$ along the [001] direction. Since the $\mathrm{Mn}-\mathrm{Mn}$ interaction in $\mathrm{CdTe}$ is known to be antiferromagnetic, the two magnetic parts of the junction are antiferromagnetically coupled via this interface engineering. The Mn layer couples antiferromagnetically also to the CrTe layers, still resulting in halfmetallic parts. The CdTe thickness can be varied so that the interaction energy is tuned to a desirable low level. In the ground state, the junction is insulating, since the spin-up states at $E_{F}$ of the two half-metallic parts are in opposite spin directions. Application of an external magnetic field can orient the moments of both half-metallic parts in parallel, so that electrons can tunnel between the spin-up bands of the halfmetallic parts, switching on the conductance. Since such a junction is free of interface states at $E_{F}$ in the half-metallic gap, its realization would constitute an ideal halfmetal-semiconductor switch.

A recent investigation [55] examined $\mathrm{CrSe} / \mathrm{MnSe}$ and $\mathrm{CrTe} / \mathrm{MnTe}$ ferromagnetic/antiferromagnetic (001) interfaces. Since MnSe and MnTe are antiferromagnetic semiconductors, while CrSe and CrTe are ferromagnets, an exchange bias is formed at the interface. The antiferromagnetism was reported to be layer-by-layer in the $\{001\}$ direction. It was found that, in the case of an uncompensated antiferromagnetic (001) interface (where the antiferromagnetic layers are perpendicular to the interface plane), a collinear half-metallic magnetic state is formed throughout the junctions, with an exchange bias of $\Delta E=-0.41 \mathrm{eV} / \mathrm{a}^{2}$ for $\mathrm{CrSe} / \mathrm{MnSe}$ and $-0.41 \mathrm{eV} / \mathrm{a}^{2}$ for $\mathrm{CrTe} / \mathrm{MnTe}$ (here, a is the in-plane lattice parameter). In the compensated (001) interface (where the antiferromagnetic layers are parallel to the interface plane), a spin-flop (non-collinear) ground state was found, with the Cr moments being perpendicular to the Mn moments; the corresponding exchange bias is $\Delta E=-0.08 \mathrm{eV} / a^{2}$ for $\mathrm{CrSe} / \mathrm{MnSe}$ and $-0.03 \mathrm{eV} / a^{2}$ for $\mathrm{CrTe} / \mathrm{MnTe}$. A similar spin-flop sate was also calculated in reference [54] in $(\mathrm{CrSe})_{1}(\mathrm{MnSe})_{1}$ and $(\mathrm{CrTe}) /(\mathrm{MnTe})$ multilayers. In view of the fragile ferromagnetism [42] and half-metallicity [66] of CrSe, we consider these conclusions rather more important for $\mathrm{CrTe} / \mathrm{MnTe}$ than for $\mathrm{CrSe} / \mathrm{MnSe}$.

\section{Effect of disorder}

The half-metallic property of zb TM-pnictides and chalcogenides is based on the hybridization gap formed by the $d$ states of the TM and the $p$ states of the $s p$ atom, and on the large exchange splitting pushing the spin-down $e_{g}$ states high in energy. Therefore, disorder (e.g., in the form of antisites or swaps) is expected to result to a loss of half-metallicity, since the $p$ - $d$ hybridization is expected to change at an impurity, and the magnetic moment of an impurity can be lower than the moment of the TM.

The energetics of $\mathrm{Cr}-\mathrm{As}$ swaps in zb-CrAs have been calculated from first principles in reference 88 for swaps up to $10 \%$. Three magnetic states were considered for the $\mathrm{Cr}_{\mathrm{As}}$ antisites ( $\mathrm{Cr}$ at the As position): ferromagnetic, antiferromagnetic, and spin-glass. It was found that the Cr-As swap is not favoured energetically in any of these cases. However, if such a swap occurs, it was found that the $\mathrm{Cr}$ antisites are antiferromagnetically coupled to the rest of the $\mathrm{Cr}$ atoms (leading to a ferrimagnetic overall picture), with an energy gain of about $2 \mathrm{eV}$ per $\mathrm{Cr}$ antisite compared to the ferromagnetic swap-state; the spin-glass energy was still higher. Furthermore, it was found that the $\mathrm{Cr}$ antisites introduce states at $E_{F}$ within the half-metallic gap, 
destroying the half-metallic property.

In another study [80, the mixing energy of As antisites in $\mathrm{zb}\left(\mathrm{Cr}_{1-x} \mathrm{As}_{x}\right) \mathrm{As}$ was calculated, with respect to the phase separated $\mathrm{zb} \mathrm{CrAs}$ and pure As. It was found that, if As is in the ground-state trigonal structure $(\alpha-\mathrm{As})$, phase separation is favoured leading to clean zb CrAs. If, however, As is in the diamond structure (which could be stabilized by a GaAs substrate), the formation of $\mathrm{As}_{\mathrm{Cr}}$ antisites is favoured for $x \approx 0.6$. In the presence of $\mathrm{As}_{\mathrm{Cr}}$ antisites, it was found that spin-down states are introduced at $E_{F}$, and half-metallicity is lost.

Furthermore, the effect of antisites on the density of states and on the magnetic moments was studied in reference [89] for $\mathrm{CrAs}, \mathrm{CrSb}, \mathrm{CrSe}, \mathrm{CrTe}$, VAs, and MnAs. Alloys of the type $\mathrm{Cr}_{1+x} \mathrm{As}_{1-x}$, for $-0.5<x<0.5$, (and similarly for the other compounds) were calculated. Notably, it was found that half-metallicity is possible in some disordered cases. $\mathrm{Mn}_{1+x} \mathrm{As}_{1-x}$, for $x>0$, remained half-metallic, with an increase of the magnetic moment (the $\mathrm{Mn}_{\mathrm{As}}$ antisites were found to align ferromagnetically to the rest of the Mn atoms). Also in all $\mathrm{Cr}$ compounds, for $x>0$, the half-metallic character is kept (for not-too-high values of $x$ ), while the magnetic moment dropped (the $\mathrm{Cr}$ antisites were found to align antiferromagnetically to the rest of the $\mathrm{Cr}$ atoms).

\section{Other tetrahedrally bonded structures}

\subsection{TM-based wurtzite compounds}

The tetrahedral environment in zb TM pnictides and chalcogenides is the essential factor to the formation of $p$ - $d$ hybrids and the gap. Therefore, one expects that TM pnictides and chalcogenides in the wurtzite structure (wz), which provides an almost tetrahedral environment, should also be half-metallic. Based on these ideas, Xie et al. 87. made a systematic theoretical study of such wz compounds, including MnSb, $\mathrm{CrAs}, \mathrm{CrSb}, \mathrm{VAs}, \mathrm{VSb}, \mathrm{CrSe}, \mathrm{CrTe}$, VSe, VTe. They concluded that half-metallicity should be present also for the wz structure. The density of states is very similar to the one in the zb structure; in the energy bands there is a degeneracy lifting at the Brillouin zone center, since there is no tetrahedral symmetry (the environment of each atom is almost, but not exactly, tetrahedral). The total moments follow the "rule of 8" (eq. (21)). Half-metallicity in wz $\mathrm{CrS}, \mathrm{CrSe}, \mathrm{CrTe}$ was also reported in reference [90]. Bismuth-based compounds in the wz structure were studied in reference [91]. VBi and $\mathrm{CrBi}$ were found to be half-metallic at their respective equilibrium lattice parameters (neglecting the strong spin-orbit coupling caused by $\mathrm{Bi}$ ), while $\mathrm{MnBi}$ was found not to be half-metallic; a lattice expansion was needed to restore half-metallicity.

Total energy calculations [61] reveal that the energy of the TM pnictides wz structure is very close to the energy of the zb structure, and the lattice constants of the two phases are almost equal. This means that the wz structure is also metastable.

\subsection{Nanoclusters and nanodots}

Nanoclusters of zb TM-pnictides and chalcogenides have also been studied theoretically [92, 93. This is interesting in view of the experimental results on MnAs nanodots 10, 21, 22, although the calculated nanoclusters are much smaller, not exceeding a size of 50 atoms (the experimental nanodots were $16 \mathrm{~nm}$ in diameter). Reference 92 presents results on free-standing nanoclusters $\mathrm{TM}_{13} \mathrm{X}_{16}$ 
with $\mathrm{TM}=(\mathrm{V}, \mathrm{Cr}, \mathrm{Mn})$ and $\mathrm{X}=(\mathrm{N}, \mathrm{P}, \mathrm{As}, \mathrm{Sb}, \mathrm{S}, \mathrm{Se}, \mathrm{Te})$. By changing the lattice constant, several ferromagnetic and antiferromagnetic magnetic configurations were arrived at (although no total energy results were reported). The $s p$ atoms at the cluster boundary were found to produce dangling bonds, affecting in some cases the halfmetallic character. When CrAs nanoclusters were embedded in a GaAs matrix, the dangling bonds were found to vanish, due to the bonding of the boundary atoms with the matrix. In reference 93 the focus was in a free-standing zb MnAs nanocluster containing 41 atoms of which 13 were Mn atoms (including a central Mn atom and a first shell of $12 \mathrm{Mn}$ atoms). Structural relaxation was taken into account. It was found that, in the ground state, the central atom couples antiferromagnetically to the first shell. The ferromagnetic state was $125 \mathrm{meV}$ higher, and half-metallic, with a gap of $1.83 \mathrm{eV}$.

\section{Recent results on the role of electron correlations and on the temperature dependence of the polarization}

Density-functional calculations, within the LSDA or GGA, can capture only a part of the electron exchange and correlation effects. In particular dynamic effects need to be described by more elaborate (and computationally heavier) extensions, such as the LSDA+DMFT (dynamical mean-field theory).

An interesting effect of dynamic electron correlations on half-metallic ferromagnets is the appearance of non-quasiparticle states (NQS) in the half-metallic gap just above $E_{F}$. These states, originating from virtual electron-magnon scattering processes, are captured within the DMFT and were calculated in reference 94 for the case of CrAs. The NQS appear at characteristic magnon energies, i.e., a few tens of meV above $E_{F}$, and can therefore affect the transport properties even at small voltages. However, to our knowledge, their exact effect on spin-polarized transport has not yet been clarified; such states should be present in all half-metallic systems, because the mechanism that brings them about is rather general 95.

Another effect of dynamic correlations can be a shifting of spectral weight towards the Fermi level, which is expected due to the form of the real part of the self-energy $\Sigma(E)$ near $E_{F}: \operatorname{Re}[\partial \Sigma / \partial E]_{E_{F}}<0$ † . This has an interesting effect on VAs, as was calculated in reference [96. Within the LSDA (or GGA), VAs is found to be a ferromagnetic semiconductor, because $E_{F}$ lies exactly between the spin-up $e_{g}$ and antibonding $t_{2 g}$ states (for spin-down it is in the half-metallic gap). The spin-up gap is tiny, of the order of $50 \mathrm{meV}$. Within the LSDA+DMFT, it is found that the spin-up $e_{g}$ and $t_{2 g}$ states are shifted towards the Fermi level, closing the gap completely and causing a transition from semiconducting to half-metallic behaviour.

We would also like to point out the problem of absence of a Fermi edge observed in the photoemission spectroscopy of ferromagnetic zb MnAs nanodots [10, 21] (see also section (2). This is in contrast to theoretical results within density-functional theory, and to our knowledge its explanation is an open problem which deserves more attention in the future.

An open question is the behaviour of the spin polarization $P$ at $E_{F}$ at elevated temperatures $T$, since it is obvious that $P$ must drop at high $T$. Only few theoretical attempts have been made in this direction [97, 98, 99, 100, examining different

$\dagger \dagger \Sigma(E)$ represents a measure of renormalization of the spectral density due to correlations; the Green function, of which the poles determine the spectrum, has the form $G(E)=\left(E-H^{0}-\Sigma(E)\right)^{-1}$, where $H^{0}$ is the Hamiltonian in the absence of electron correlations. 
mechanisms that can contribute to the drop of $P(T)$. These depend on many factors, including the exact band structure and position of $E_{F}$ in the gap, but the theoretical methods have not been applied to zb pnictides and chalcogenides. In order to obtain a quantitative result, these theories have to be refined and unified (in our opinion, this is one of the most challenging theoretical problems in the field of half-metallic ferromagnets); they indicate, however, that a high $P$ at room temperature requires (i) $T_{C}$ to be considerably higher than room temperature and (ii) $E_{F}$ to be deep in the gap, and not in the proximity of a band edge. Experimentally, $P(T)$ can be approached indirectly via transport measurements in magnetic tunnel junctions under some assumptions on the polarization-dependence of the magnetoresistance [101].

\section{Summary and outlook}

In this review we have summarized the work reported on transition metal pnictides and chalcogenides in the zinc-blende structure. Relatively few experimental results have been published. We believe that this is due to the difficulties in experimental growth of these structures. So far, experimental fabrication has been demonstrated only for ultrathin layers (including multilayers) of $\mathrm{CrAs}$ and $\mathrm{CrSb}$ and nanodots of $\mathrm{MnAs}$, and evidence exists for ultrathin layers of MnAs and $\mathrm{CrTe}$ (see section 22). Magnetic measurements have been reported, but no evidence has been shown of half-metallic behaviour (or of its absence, except for the insulating phase reported for zb MnAs nanodots [10, 21]). At the same time, there are many theoretical and computational investigations on the subject, including other related structures. To a great extent, these concern the structural stability of the zb phase (see section 5), and demonstrate that this phase can at most be grown epitaxially for a few layers (in agreement with experiments on $\mathrm{CrAs}$ and $\mathrm{CrSb}$ ). Although no prediction on future experimental results can be made, we conclude that, if zb pnictides or chalcogenides are to be used in spintronics applications (such as magnetic tunnel junctions or spin injection), it has to be done in a way that a few monolayers are sufficient.

On the other hand, from the calculated electronic structure and transport point of view, these compounds are excellent, and growth of a few monolayers is probably enough for applications. Theoretical investigations show that, at the contact with semiconductors, no interface states in the gap are present, and that the half-metallic state is also robust against tetragonal distortions. The ferromagnetic interactions are dominant even in delta-doped (i.e., one-monolayer) cases, so that even one monolayer should be enough to provide fully spin-polarized transport. Calculations including dynamic correlations show that the half-metallic property (at least in CrAs) is not affected, except for the non-quasiparticle states just above $E_{F}$.

The material with the most robust ferromagnetism is probably CrAs, which is found by calculations to have the highest Curie temperature. It is reasonable to assume that, among the materials which were reviewed here, the best candidates for future applications are $\mathrm{CrAs}$ and $\mathrm{CrSb}$ (since they were the zb pnictides fabricated so-far), or delta-doped compounds (including $\mathrm{Si}$ and $\mathrm{Ge}$ ) in the monolayer or sub-monolayer range.

\section{References}

[1] de Groot R A, Mïler F M, van Engen P G, and Buschow K H J 1983 Phys. Rev. Lett. 502024

[2] Akinaga H, Manago T, and Shirai M 2000 Japan. J. Appl. Phys. 39 L1118 
[3] Watanabe H, Kazama N, Yamaguchi Y, Ohashi M 1969 J. Appl. Phys. 40 1128; Boller H and Kallel A 1971 Solid State Commun. 91699

[4] Kusakabe K, Geshi M, Tsukamoto H, and Suzuki N 2004 J. Phys.: Condens. Matter 16 S5639

[5] Sieberer M, Redinger J, Khmelevskyi S, and Mohn P 2006 Phys. Rev. B 73024404

[6] Yao K L, Jiang J L, Liu Z L, and Gao G Y 2006 Phys. Lett. A 359326

[7] Mizuguchi, M, Akinaga, H, Manago, T, Ono, K, Oshima, M, and Shirai M 2002 J. Magn. Magn. Mater. 239269

[8] Mizuguchi M, Ono K, Oshima M, Okabayashi J, Akinaga H, Manago T, and Shirai M 2002 Surface Review and Letters $\mathbf{9} 331$

[9] Mizuguchi M, Akinaga H, Manago T, Ono K, Oshima M, Shirai M, Yuri M, Lin HJ, Hsieh HH, Chen CT 2002 J. Appl. Phys. 917917

[10] Oshima M, Ono K, Mizuguchi M, Yamada M, Okabayashi J, Fujimori A, Akinaga H, and Shirai M 2002 Int. J. Mod. Phys. B 161681

[11] Ofuchi H, Mizuguchi M, Ono K, Oshima M, Akinaga H, and Manago T 2003 Nucl. Instrum. Methods B 199227

[12] Shirai M 2001 Physica E 10143

[13] Bi J F, Zhao J H, Deng J J, Zheng Y H, Li S S, Wu X G, Jia Q J 2006 Appl. Phys. Lett. 88 142509

[14] Akinaga H and Mizuguchi M 2004 J. Phys.: Condens. Matter 16 S5549

[15] Zhao J H, Matsukura F, Takamura K, Abe E, Chiba D, and Ohno H 2001 Appl. Phys. Lett. 792776

[16] Deng J J, Zhao J H, Bi J F, Niu Z C, Yang F H, Wu X G, and Zheng H Z 2006 J. Appl. Phys. 99093902

[17] Shirai M, Ogawa T, Kitagawa I, and Suzuki N 1998 J. Magn. Magn. Mater. 177-181 1383

[18] Liu B G 2003 Phys. Rev. B 67172411

[19] Galanakis I and Mavropoulos P 2003 Phys. Rev. B 67104417

[20] Zhao J H, Matsukura F, Takamura K, Chiba D, Ohno Y, Ohtani K, and Ohno H 2003 Materials Science in Semiconductors Processing 6507

[21] Ono K, Okabayashi J, Mizuguchi M, Oshima M, Fujimori A, and Akinaga H 2002 J. Appl. Phys. 918088

[22] Okabayashi J, Mizuguchi M, Ono K, Oshima M, Fujimori A, Kuramochi H, and Akinaga H 2004 Phys. Rev. B 70233305

[23] Kawakami R K, Johnston-Halperin E, Chen L F, Hanson M, Guebels N, Speck J S, Gossard A C, and Awschalom D D 2000 Appl. Phys. Lett. 772379

[24] Tanaka M 2005 J. Cryst. Growth 27825

[25] Choi J Y, Choi J Y, Choi S Y, Hong S C, and Cho S L 2005 Journal of the Korean Physical Society $47 \mathrm{~S} 497$

[26] Sreenivasan M G, Hou X J, Teo K L, Jalil M B A, Liew T, and Chong T C 2006 Thin Solid Films 505133

[27] Wei S-H and Zunger A 1987 Phys. Rev. B 37 8958; Wei S-H and Zunger A 1987 Phys. Rev. B 352340

[28] Akai H 1998 Phys. Rev. Lett. 81 3002; Sato K, Dederichs P H, Katayama-Yoshida H, and Kudrnovský 2004 J. Phys.: Condens. Matter 16 S5491

[29] Zhang M, Hu H, Liu G, Liu Z, Cui Y, and Wu G 2004 J. Low Temp. Phys. 135267

[30] Yao K L, Gao G Y, Liu Z L, and Zhu L 2005 Solid State Commun. 133301

[31] Sakuma A 2002 J. Phys. Soc. Japan 712534

[32] Xu Y Q, Liu B G, and Pettifor D G 2002 Phys. Rev. B 66184435

[33] Shirai M 2003 J. Appl. Phys. 936844

[34] Pask J E, Yang L H, Fong C Y, Pickett W E, and Dag S 2003 Phys. Rev. 67224420

[35] Xie W H, Xu Y Q, Liu B G, and Pettifor D G 2003 Phys. Rev. Lett. 91 037204; idem Phys. Rev. Lett. 91219901 (erratum)

[36] Xie W H and Liu BG 2003 J. Phys.: Condens. Matter 155085

[37] Sanyal B, Bergqvist L, and Eriksson O 2003 Phys. Rev. B 68054417

[38] Zhang M, Hu H, Liu G, Cui Y, Liu Z, Wang J, Wu G, Zhang X, Yan L, Liu H, Meng F, Qu J and Li Y 2003 J. Phys.: Condens. Matter 155017

[39] Zhang M, Hu H N, Liu Z H, Liu G D, Cui Y T, and Wu G H 2004 J. Magn. Magn. Mater. $\mathbf{2 7 0} 32$

[40] Galanakis I, Dederichs P H, and Papanikolaou N 2002 Phys. Rev. B 66 134428; Galanakis I, Dederichs P H, and Papanikolaou N 2002 Phys. Rev. B 66174429

[41] Fong C Y, Qian M C, Pask J E, Yang L H, and Dag S 2004 Appl. Phys. Lett. 84239

[42] Sasioglu E, Galanakis I, Sandratskii L M, and Bruno P 2005 J. Phys.: Condens. Matter 17 
3915

[43] Liu G Q and Liu B G 2006 Phys. Rev. B 73045209

[44] Qian M C, Fong C Y, Liu K, Pickett W E, Pask J E, and Yang L H 2006 Phys. Rev. Lett. 96 027211

[45] Qian M C, Fong C Y, and Yang L H 2004 Phys. Rev. B 70052404

[46] Dag S, Tongay S, Yildirim T, Durgun E, Senger R T, Fong C Y, and Ciraci S 2005 Phys. Rev. B 72155444

[47] Mavropoulos P, Sato K, Zeller R, Dederichs P H, Popescu V, and Ebert H 2004 Phys. Rev. B 69054424

[48] Shirai M, Ikeuchi K, Taguchi H and Akinaga H 2003 J. Supercond. 1627

[49] Zheng J C and Davenport J W 2004 Phys. Rev. B 69144415

[50] Zhao Y-J, Geng W T, Freeman A J, and Delley B 2002 Phys. Rev. B 65113202

[51] Kübler J 2003 Phys. Rev. B 67220403

[52] Kim M and Freeman A J 2004 Appl. Phys. Lett. 854983

[53] Miao M S and Lambrecht W R L 2005 Phys. Rev. B 71214405

[54] Nakamura K, Ito T, and Freeman A J 2005 Phys. Rev. B 72064449

[55] Nakamura K, Kato Y, Akiyama T, Ito T, and Freeman A J 2006 Phys. Rev. Lett. 96047206

[56] Sanyal B, Eriksson O, and Aron C 2006 Phys. Rev. B (to be published)

[57] Asada T and Terakura K 1993 Phys. Rev. B 4715992

[58] Liechtenstein A I, Katsnelson M I, Antropov V P, and Gubanov V A 1987 J. Magn. Magn. Mater. 6765

[59] Sanvito S and Hill N A 2000 Phys. Rev. B 6215553

[60] Continenza A, Picozzi S, Geng W T, and Freeman A J 2001 Phys. Rev. B 64085204

[61] Miao M S and Lambrecht W R L 2005 Phys. Rev. B 71064407

[62] Zhao Y J and Zunger A 2005 Phys. Rev. B 71132403

[63] Lambrecht W R L, Miao M S, and Lukashev P 2005 J. Appl. Phys. 97 10D306

[64] Yamana K, Geshi M, Tsukamoto H, Uchida I, Shirai M, Kusakabe K, and Suzuki N 2004 J. Phys.: Condens. Matter 16 S5815

[65] Shi L J and Liu B G 2005 J. Phys.: Condens. Matter 171209

[66] Miao M S and Lambrecht W R L 2005 Phys. Rev. B 72064409

[67] 39 of 138 Miao MS and Lambrecht WRL 2005 J. Appl. Phys. 97 10C304

[68] Mavropoulos P, Galanakis I, and Dederichs P H 2004 J. Phys.: Condens. Matter 164261

[69] Jenkins S J 2004 Phys. Rev. B 70 245401; Jenkins S J and King D A 2002 Surf. Sci 501 L185; idem 2001 Surf. Sci 494 L793; Ležaić M, Galanakis I, Bihlmayer G, and Blügel S 2005 J. Phys.: Condens. Matter 17 3121; Galanakis I 2002 J. Phys.: Condens. Matter 146329

[70] Galanakis I 2002 Phys. Rev. B 66012406

[71] Kang B S, Oh S K, Chung J S, and Kang H J 2005 Thin Solid Films 488204

[72] Byun Y, Lee J I, and Jang Y R 2006 J. Appl. Phys. 99 08J101

[73] Qian M C, Fong C Y, Pickett W E, Pask J E, Yang L H, and Dag S 2005 Phys. Rev. B 71 012414

[74] Lee JI, Byun Y, and Jang YR 2006 Surf. Sci. 6001608

[75] Lee J I and Hong S C 2006 IEEE Transactions on Magnetics 422936

[76] Mavropoulos P, Ležaić M, and Blugel S 2005 Phys. Rev. B 72174428

[77] Attema J J, de Wijs G A, and de Groot R A 2006 J. Phys. D: Appl. Phys. 39 793; Nagao K, Miura Y, and Shirai M 2006 Phys. Rev. B 73 104447; Galanakis G, Ležaić M, Bihlmayer G, and Blügel S 2005 Phys. Rev. B 71 214431; Galanakis I 2004 J. Phys.: Condens. Matter 16 8007

[78] Shirai M 2004 J. Phys.: Condens. Matter 16 S5525

[79] Nagao K, Shirai M, and Miura Y 2004 J. Appl. Phys. 956518

[80] Bengone O, Eriksson O, Fransson J, Turek I, Kudrnovsky J, and Drchal V 2004 Phys. Rev. B 70035302

[81] Cha G B, Cho S G, and Hong S C 2004 Phys. Status Solidi (b) 2411423

[82] Fong C Y and Qian M C 2004 J. Phys.: Condens. Matter 16 S5669

[83] Wu RQ, Liu L, Peng GW and Feng YP 2006 J. Appl. Phys. 99093703

[84] Sanvito S and Hill NA 2001 Phys. Rev. Lett. 87267202

[85] Continenza A, Antoniella F, and Picozzi S 2004 Phys. Rev. B 70035310

[86] Picozzi S, Ležaić M, and Blügel S 2006 Phys. Status Solidi (a) 2032738

[87] Xie W H, Liu B G, and Pettifor D G 2003 Phys. Rev. B 68134407

[88] Shirai M, Seike M, Sato K and Katayama-Yoshida H 2004 J. Magn. Magn. Mater. 272-276 344

[89] Galanakis I, Özdog̃an K, Şaşıoglu E, and Aktas B 2006 Phys. Rev. B 74 140408(R) 
[90] Zhang M, Bruck E, de Boer F R, Liu G D, Hu H N, Liu Z H, Cui Y T, and Wu G C $2004 J$. Mater. Res. 192738

[91] Zhang M, Bruck E, de Boer F R, and Wu G H 2005 J. Appl. Phys. 97 10C306

[92] Nakao M 2004 Phys. Rev. B 69214429

[93] Qian M C, Fong C Y, Pickett W E, and Wang H Y 2004 J. Appl. Phys. 957459

[94] Chioncel L, Katsnelson M I, de Wijs G A, de Groot R A, and Lichtenstein A I 2005 Phys. Rev. B 71085111

[95] Irkhin V Y and Katsnelson M I 2002 Eur. Phys. J. B 30481

[96] Chioncel L, Mavropoulos P, Ležaić M, Blügel S, Arrigoni E, Katsnelson M I, and Lichtenstein A I 2006 Phys. Rev. Lett. 96197203

[97] Itoh H, Ohsawa T, and Inoue J 2000 Phys. Rev. Lett. 842501

[98] Skomski R and Dowben PA 2002 Europhys. Lett. 58544

[99] Chioncel L, Arrigoni E, Katsnelson M I, and Lichtenstein A I 2006 Phys. Rev. Lett. 96137203

[100] Ležaić M, Mavropoulos P, Enkovaara J, Bihlmayer G, and Blügel S 2006 Phys. Rev. Lett. 97 026404

[101] Garcia V, Bibes M, Barthélémy A, Bowen M, Jacquet E, Contour J-P, and Fert A 2004 Phys. Rev. B 69052403

[102] Rader O, Ležaić M, Blügel S, Fujimori A, Kimura A, Kamakura N, Kakizaki A, Miyanishi S, and Akinaga H 2005 New J. Phys. 7111 